ment of liver fibrosis. The FibroScan ${ }^{\circledR}$ device (Echosens, Paris, France) functions by transmitting low-frequency vibrations directly into hepatic tissue, these generate an elastic shear wave that travels through the tissue at a rate that depends on its stiffness: the stiffer the tissue the faster the wave travels. The wave velocity is detected by ultrasonography.

Foucher and colleagues have carried out a prospective study of the accuracy of this new device in a cohort of 711 patients with liver disease of various etiologies; 354 of these patients underwent liver biopsy after the elastography procedure.

In comparison with histologic findings, liver stiffness was found to correlate significantly with the fibrotic stage $(P<0.0001)$. Positive and negative predictive values for the diagnosis of cirrhosis were $91 \%$ and $92 \%$, respectively, with an elastography stiffness cut-off value set at $17.6 \mathrm{kPa}$ to indicate cirrhosis. Marked associations were also identified between liver stiffness and clinical, biological, and morphological parameters of liver disease.

The authors conclude that transient elastography shows promise for noninvasive detection of cirrhosis and for assessing its severity, irrespective of the etiology of the liver disease.

Katy Cherry

Original article Foucher J et al. (2006) Diagnosis of cirrhosis by transient elastography (FibroScan): a prospective study. Gut 55: 403-408

\section{Outcome of liver donors after right hepatectomy}

Living donor liver transplantation has increased tremendously in popularity since the technique was introduced in 1996, partly because rightlobe hepatectomy allows large grafts to be obtained-up to two-thirds of the donor's liver volume-that make adult-to-adult transplantation feasible. The benefits of large graft volumes are obvious for the recipient, but the long-term sequelae for the donor are still unknown.

Cho et al. transplanted right-lobe grafts without the middle hepatic vein, and found that over the 12 months after surgery, the outcome of 74 donors whose remnant liver volume was $<35 \%$ of their original liver volume as assessed by CT was no poorer than that of 72 donors whose remnant liver volume was $\geq 35 \%$. Adequate safety margins would be provided by a lower limit for remnant liver volume of $30 \%$, they suggest.

Chan et al. transplanted right-lobe grafts including the middle hepatic vein, and found that at long-term follow-up (a median of 47.4 months after surgery) the 29 donors' regenerated livers were measurably (although not statistically significantly) smaller than their preoperative volume, as assessed by CT. Interestingly, there was an inverse relationship with remnant liver volume: donors with the biggest liver remnant after hepatectomy had the greatest regeneration deficit.

Even minor donor morbidities should not be ignored, say Cho et al., because a second insult in the setting of hepatic insufficiency after liver donation could initiate a potentially fatal cycle of complications.

Caroline Barranco

Original articles Cho JY et al. (2006) Outcome of donors with a remnant liver volume of less than 35\% after right hepatectomy. Liver Transpl 12: 201-206

Chan SC et al. (2006) Long-term biological consequences of donor right hepatectomy including the middle hepatic vein in adult-to-adult live donor liver transplantation. Liver Transpl 12: 259-263

\section{Dietary interventions and colorectal cancer risk}

Observational studies have suggested that low-fat eating patterns are associated with a decreased risk of colorectal cancer, and similar findings have also been recorded with calcium plus vitamin D supplementation. Primary prevention trials are lacking, however, despite colorectal cancer being the second leading cause of death from cancer in the US. Two randomized clinical trials have now been carried out to investigate both dietary intake and vitamin and mineral supplements in the setting of colorectal cancer, using participants recruited from 40 clinical centers involved in the Women's Health Initiative (WHI), which was established by the NIH in 1991 to assess causes of death in postmenopausal women.

The WHI Dietary Modification Trial recruited 48,835 women, randomly assigned to a low-fat dietary intervention group $(n=19,541)$ or a control group $(n=29,294)$. Members of the intervention group underwent an intensive behavioral modification program that aimed to reduce their 